\title{
Usages de la sexualité dans la clinique du transsexualisme
}

Laurence Hérault, Université d’Aix-Marseille, Idemec.

Aborder la question de la sexualité dans le cadre du transsexualisme, c'est un peu s’intéresser à une éminence grise. Au premier abord, sa place et sa fonction semblent insignifiantes dans la caractérisation de ce trouble tout entier centré sur les problèmes d'identité sexuée. En même temps, un esprit attentif ne peut manquer d'être intrigué par sa présence à la fois discrète et régulière et ne peut que s'interroger sur son rôle effectif en ce domaine. Cette situation intrigante est perceptible dans la description que le DSM fait de ce trouble. Ce manuel psychiatrique de référence inscrit, en effet, la dysphorie de genre parmi les troubles sexuels et lui fait côtoyer, notamment, les paraphilies. Pourtant les critères diagnostiques n'évoquent aucunement la sexualité des sujets puisqu'être atteint d'un tel trouble suppose une identification intense et persistante à l'autre sexe, un sentiment persistant d'inconfort par rapport à son sexe, aucun problème d'intersexuation et une souffrance cliniquement significative. Si le transsexuel est troublé, ce n'est donc pas en raison d'un dysfonctionnement ou d'une déviation d'ordre sexuel, la chose semble entendue. Toutefois, à la fin de la description, il est recommandé de spécifier si le sujet est attiré sexuellement par les hommes, par les femmes, par les deux sexes, ni par un sexe ni par l'autre, sans qu'il soit dit ce que l'on doit faire d'une telle précision. Au regard de cette présence modeste mais constante, il ne semble pas inutile de se demander à quoi sert la sexualité des personnes transsexuelles ${ }^{1}$. Quel est son rôle dans la caractérisation du transsexualisme et dans son approche clinique? C'est ce que nous allons essayer de débrouiller ici dans l'intention d'interroger, de manière générale, les liens qui unissent genre et sexualité dans notre société.

\section{Le transsexualisme ou la marginalisation de la sexualité}

Le transsexualisme comme catégorie nosographique apparaît en 1949 dans l'article d'un psychiatre-sexologue américain, David Cauldwell. En référence à Krafft-Ebing, il choisit le terme psychopathia transsexualis pour décrire le cas d'Earl qui souhaite qu'un chirurgien lui enlève les seins et les ovaires et lui construise un pénis artificiel. Cauldwell définit les transsexuels comme des individus qui souhaitent être membres du sexe auquel ils n’appartiennent pas et qui désirent modifier chirurgicalement leurs caractéristiques physiques pour qu'elles ressemblent à celles de l'autre sexe. Dans un ouvrage publié en 1950, il précise :

\footnotetext{
${ }^{1}$ Par souci de cohérence, j’utiliserai, la plupart du temps, la manière médicale de désigner les personnes transsexuelles qui se réfère à leur sexe d'origine. Dans le vocabulaire de la psychiatrie et de la sexologie, les termes «transsexualisme masculin » et "transsexuel » qualifient ainsi les personnes $\mathrm{MtF}$ (male to female); les termes « transsexualisme féminin » et «transsexuelle» les FtM (female to male). Il m'arrivera cependant d'utiliser aussi un vocabulaire non-médical mais dans ce cas j'utiliserai les expressions "femmes transgenres » pour désigner les $\mathrm{MtF}$ et « hommes transgenres » pour désigner les FtM afin d'éviter toute confusion pour le lecteur.
} 
«trans-sexuals are individuals who are physically of one sex and apparently psychologically of the opposite sex. Trans-sexuals include heterosexuals, homosexuals, bisexuals and others. A large element of transvestites have trans-sexual leanings. " . Cette caractérisation initiale est intéressante car elle montre bien la manière dont se redécoupe, alors, le champ de l'inversion sexuelle formalisée au siècle précédent. Elle participe, en effet, d'un mouvement de conceptualisation qui trace de nouvelles frontières entre des "pathologies » plus ou moins associées auparavant dans le cadre de l'inversion, comme l'homosexualité, le transvestisme, le transsexualisme et même l'hermaphrodisme. On voit bien, en outre, qu'en indiquant trouver chez les transsexuels l'ensemble des orientations sexuelles répertoriées, Cauldwell affirme que le transsexualisme n'est pas un problème de sexualité : le comportement sexuel ne spécifie pas les transsexuels, il n'est pas à considérer comme un symptôme du transsexualisme. Cette position est aussi celle d'Harry Benjamin qui est le véritable promoteur du transsexualisme à partir des années 1950. Dans son ouvrage, le phénomène transsexuel, publié en 1966, sa caractérisation du transsexualisme se constitue essentiellement par rapport au transvestisme mais elle trace également les frontières entre transvestisme, transsexualisme et homosexualité. Selon lui, si par un certain nombre de traits superficiels on peut être tenté d'assimiler homosexuels, transvestis et transsexuels, leurs positionnements sexuel et sexué invitent à les considérer comme des personnes différentes. Leur problème respectif est clairement différenciable : «To bring the discussion regarding the three deviations (...), a nutshell characterization would be this: The transvestite has a social problem. The transsexual has a gender problem. The homosexual has a sex problem. ».

Cette conceptualisation " initiale » du transsexualisme est celle qui va dominer le développement de la clinique transsexuelle et c'est d'ailleurs celle que l'on retrouve dans la caractérisation donnée par le DSM. Les affinements ou les remaniements qui verront le jour dans les années 1970 ne modifieront pas l'affirmation selon laquelle le transsexualisme est un problème de genre et non de sexualité. Norman Fisk, par exemple, ne la remet pas en question lorsqu'il propose en 1973 de requalifier le transsexualisme en dysphorie de genre. Ce syndrome, qui qualifie toutes les personnes qui expérimentent un inconfort quant à leur genre d'assignation, rend assez floues les frontières nosologiques que ses prédécesseurs s'étaient efforcés de tracer puisqu'on peut y inclure un certain nombre de ceux que l'inversion sexuelle rassemblait autrefois. Cependant, il s'affirme clairement comme une « pathologie du genre » : ce qui lie l'ensemble des dysphoriques ainsi étiquetés c'est bien un problème d'identité sexuée. Dans une toute autre optique, cette focalisation sur le genre est également visible chez Stoller qui propose, quant à lui, de distinguer plus précisément les demandeurs, reconnaissant parmi eux, des «transsexuels vrais » ou "transsexuels primaires » et des «pseudotranssexuels» ou "transsexuels secondaires». Les premiers ont, depuis leur enfance, le sentiment d'appartenir au sexe opposé, ce qui les a conduit assez souvent au travestissement (sans connotations sexuelles "fétichistes ») et les amène à demander précocement une réassignation (à la fin de l'adolescence ou au début de l'âge adulte). Les «transsexuels secondaires ", en revanche, expriment tardivement leur souhait de transformation ; ils se sont également travestis mais seulement à partir de l'adolescence et ce travestissement pouvait avoir une motivation sexuelle. Ils ont tenté le plus souvent d'ignorer leur inclination et ont pu se conformer, durant une partie de leur vie, au rôle associé à leur genre d'assignation et beaucoup ont été mariés et sont devenus parents. Pour Stoller, la demande de réassignation renvoie, chez eux, à des configurations psychiques ou pathologiques distinctes du véritable transsexualisme qui peuvent être mieux traitées par la psychothérapie. Autrement dit, le transsexualisme authentique est essentiellement un problème d'identité de genre dans lequel le motif sexuel est secondaire ou, du moins, non symptomatique. Quant au transsexualisme 
secondaire, on perçoit que la sexualité y tient une place plus prégnante même si elle n’est pas nécessairement centrale.

Cette focalisation consensuelle sur le genre n'empêche, cependant, pas un usage particulier de la sexualité des personnes concernées. Historiquement cet usage s'est imposé à la fois pour des raisons théoriques et stratégiques. Revenons à Benjamin pour comprendre le rôle effectif de la sexualité et, plus particulièrement, de l'orientation sexuelle dans la clinique du transsexualisme. Dans le phénomène transsexuel, Benjamin ne se contente pas de différencier les troubles, il s'attache aussi à qualifier précisément l'orientation sexuelle des transsexuels : "From all that has been said, it seems evident that the question "Is the transsexual homosexual?" must be answered "yes" and "no". "Yes", if his anatomy is considered; "no" if his psyche is given preference. What would be the situation after corrective surgery has been performed and the sex anatomy now resembles that of a woman? Is the "new woman" still a homosexual man? "Yes", if pedantry and technicalities prevail. "No" if reason and common sense are applied and if the respective patient is treated as an individual and not as a rubber stamp” (Benjamin, 1996, chap. 2). S'il n'est guère difficile de souscrire à l'argument de Benjamin selon lequel une femme transgenre opérée, attirée par les hommes, est hétérosexuelle, on ne peut s'empêcher, en même temps, de remarquer qu'il n'évoque pas la situation inverse, à savoir l'intérêt éventuel des MtF pour des partenaires de sexe féminin. Il semble, au fond, que les MtF seraient attirées uniquement par les hommes (et les FtM par les femmes), c'est-à-dire que les transsexuels seraient fondamentalement hétérosexuels (au regard du genre revendiqué) et la transition viendrait rendre visible cette hétérosexualité essentielle. Benjamin est pourtant, dès le début, confronté à la diversité des orientations sexuelles des patients qu'il reçoit. Schaefer et Wheeler montrent, par exemple, que parmi les dix premiers patients de Benjamin, il est possible de reconnaître, à l'aide de l'échelle de Kinsey (qui conjugue le degré d'exclusivité et le genre des partenaires désirés), deux hétérosexuels, cinq bisexuels et trois homosexuels. Ekins montre que cette "réalité clinique » a tendance à disparaître dans les travaux ultérieurs de Benjamin où il affine la conceptualisation du transsexualisme et dans lesquels il marginalise et normalise la sexualité des transsexuels. A partir du milieu des années 1950, il va non seulement affirmer avec force que leur sexualité n'est pas un symptôme du transsexualisme mais suggérer aussi qu'ils sont invariablement homosexuels (du point de vue de leur sexe d'origine), c'est-à-dire hétérosexuels (du point de vue de leur sexe revendiqué). Le processus de marginalisation tient évidemment, comme on l'a vu, à une option théorique et nosologique qui souhaite " enfin » distinguer les «troubles de la sexualité » des «troubles du genre ». En revanche, il semble que la normalisation de l'orientation sexuelle des transsexuels renvoie à une stratégie d'ordre plus pragmatique qui tient, d'une part, à la recevabilité d'une spécialité médicale nouvelle, et d'autre part à la mise en place des protocoles de soins. Ekins suggère, en effet, que cette « hétérosexualisation » des patients, a vraisemblablement permis de rendre plus respectable et légitime les traitements hormono-chirurgicaux et a favorisé leur développement dans un contexte médical et social tout à la fois ouvert et réticent. Mais elle a aussi affaire avec la manière même, dont se sont progressivement constitués les protocoles de traitement. Si la demande de transformation figurait au titre de symptôme essentiel du transsexualisme, aucune équipe médicale n'était cependant prête à opérer les nombreux demandeurs qui les consultaient dans ce but. D'une certaine manière, les critères de sélection pour la réassignation allaient progressivement prendre le pas sur les critères diagnostiques. Pour autoriser l'opération d'un patient il ne s'agissait pas seulement de savoir s'il s'identifiait à l'autre sexe et formulait une demande de réassignation, mais il fallait aussi s'inquiéter des chances de réussite de la transformation afin d'éviter les regrets post-opératoires, de prévenir les poursuites judiciaires des mécontents et de contrer les accusations de charlatanisme. 
Comme le montre J. Meyerowitz (2002), les protocoles qui vont être progressivement mis en place s’attacheront à développer une véritable évaluation du patient, de sa situation et de son projet $^{2}$. Ils insisteront, notamment, sur l'évaluation psychologique du candidat pour s'assurer de la permanence de l'identification dans l'autre sexe et de l'absence de maladie mentale sévère; sur la nécessité d'une démarche de transition progressive (vivre préalablement quelques mois/années dans le genre souhaité et prendre des hormones avant d'accéder à la chirurgie); sur l'évaluation des capacités du candidat à s’inscrire adéquatement dans son nouveau genre et à mener à bien son projet de transition (conscience des possibilités et des limites de la chirurgie, capacités d'insertion professionnelle et sociale, mais aussi respect des conventions sexuées et sexuelles). Autrement dit, la sexualité, sans faire explicitement partie des critères diagnostiques du transsexualisme, acquiert, dès les premiers protocoles, une certaine importance par le biais des critères sélectifs permettant l'accès au traitement: un candidat qui envisageait, après la transition, une relation hétérosexuelle, voire même une vie maritale, avait un meilleur profil compte-tenu des conventions sexuelles médicales ${ }^{3}$ et sociales. Bien évidemment, les candidats à la transition confrontés à cette sélection, ont progressivement appris à optimiser leur chance et à répondre adéquatement aux exigences posées : les histoires de vie et les projets ont ainsi eu tendance à se typifier, ce qui a eu, entre autres, pour effet « d'invisibiliser » plus encore la diversité des orientations et des expériences sexuelles. Les orientations non-hétérosexuelles ne disparaissent cependant pas totalement du tableau, mais elles sont assez généralement considérées comme problématiques. Ceux qui, comme Stoller, tiennent à une spécification rigoureuse du transsexualisme, ont tendance à considérer l'hétérosexualité pré-opératoire comme un indice de transvestisme ou de transsexualisme secondaire: si un consultant tardif a expérimenté une vie hétérosexuelle relativement longue, éventuellement épanouie et féconde, il y a tout lieu d'ajourner sa demande. Quant à ceux qui se réfèrent plus volontiers à la catégorie nosologique extensive de la dysphorie de genre, l'hétérosexualité pré-opératoire n'est pas, pour eux, un critère d'exclusion mais figure le plus souvent parmi les « facteurs prédictifs négatifs », c'est-à-dire ceux qui sont susceptibles d'entraîner des regrets, des troubles dépressifs ou encore des comportements suicidaires chez le patient réassigné. Autrement dit, alors même que la sexualité n'est pas un symptôme du transsexualisme, qui est très majoritairement considéré comme une pathologie de l'identité sexuée, elle intervient tout de même pour orienter les décisions diagnostiques et thérapeutiques. On peut noter également, comme le fait Ekins (2005) une tendance hétéronormative à l'œuvre dans l'évaluation des candidats. Leur hétérosexualité (au regard du sexe revendiqué) devient ainsi, assez souvent, une sorte de « preuve » d'authenticité de leur identité masculine (pour les FtM) ou féminine (pour les MtF) fondamentale. Finalement, la transition est envisagée implicitement comme une procédure de révélation d'une «hétérosexualité masquée » et il semble plus difficile d'admettre qu'elle puisse mener à l'homosexualité. La "présence marginale » de l'orientation sexuelle dans la description du DSM commence ainsi à s'éclaircir, mais il nous reste à évoquer une approche alternative, élaborée dans les années 1980, pour bien mesurer la complexité de son rôle dans le transsexualisme.

\footnotetext{
${ }^{2}$ Les protocoles contemporains privilégient d'ailleurs, pour la plupart, des procédures d'évaluation semblables.

${ }^{3}$ Rappelons que l'homosexualité est une pathologie psychiatrique répertoriée dans le DSM jusque dans les années 1970. Son retrait n’en a été total que dans la troisième version révisée (la première version du DSM III, mentionne l'homosexualité ego-dystonique qui est toujours présente dans la plupart des manuels psychiatriques français actuels).
} 


\section{L’autogynéphilie ou la sexualisation du transsexualisme}

Dans le courant des années 1980, le sexologue Ray Blanchard a inventé le terme autogynéphilie pour décrire l'excitation sexuelle que certains hommes éprouvent à se penser ou s’imager en femmes. Il s'est personnellement intéressé à cette " orientation érotique ", via les récits des patients qu'il recevait alors à la "Gender Identity Clinic » de Toronto. Cet intérêt sexuel masculin lié à l'image féminine de soi était, cependant, loin d'être inconnu des psychiatres et des sexologues puisque on en trouvait mention dans la littérature clinique depuis le début du siècle. En créant l'autogynéphilie, Blanchard n'a, cependant, pas pour seule ambition de contribuer à la rénovation lexicale de certains troubles de la sexualité déjà référencés. Son projet a une visée plus théorique : par l'intermédiaire de l'autogynéphilie, il souhaite proposer une clarification du transsexualisme, c'est-à-dire élaborer une nouvelle typologie des troubles de l'identité de genre . Le travail d'investigation entamé par l'un de ses maîtres en sexologie, Kurt Freund, fonde son projet. Ce dernier, sur la base d'une étude sur la "male cross-gender identity », montre que celle-ci se développe différemment chez les homosexuels et les hétérosexuels: l'affirmation précoce d'une identité féminine est caractéristique chez les premiers et le travestissement fétichiste chez les seconds. Il en conclut à l'existence de deux types distincts de transsexualisme, l'un homosexuel et l'autre hétérosexuel, et affirme, bien qu'avec une certaine prudence, l'importance de la sexualité dans ce trouble. C'est ce lien postulé entre troubles de la sexualité et troubles du genre qui va orienter la recherche de Blanchard. A partir du milieu des années 1980, il mène, une série d'enquêtes quantitatives portant sur des hommes biologiques consultant pour dysphorie de genre et distingués selon leur orientation sexuelle (celle-ci étant définie à l'aide de deux échelles mesurant respectivement le degré d'androphilie et de gynéphilie des sujets et exprimée relativement à leur sexe biologique). Les résultats indiquent que les transsexuels hétérosexuels (gynéphiles), bisexuels et asexuels présentent des caractéristiques semblables (proches de celles des transvestis) qui les distinguent statistiquement des transsexuels homosexuels (androphiles). Ces derniers développent une identité féminine très précoce ; ils rapportent rarement des épisodes de travestissement à dimension érotique ; l'image d'euxmêmes en femmes ne suscite généralement pas d'excitation sexuelle ; enfin, ils demandent une réassignation alors qu'ils sont encore jeunes. Les autres transsexuels MtF sont moins féminins dans l'enfance ; leur travestissement est très souvent érotisé et ils mentionnent fréquemment des fantasmes ou des épisodes autogynéphiliques ; ils sont généralement plus âgés lors de la demande de réassignation .

D’une certaine manière, ces résultats ne sont pas vraiment originaux : on reconnaît, grosso modo, dans les profils ainsi établis les transsexuels primaires et secondaires déjà référencés. Ce qui différencie cependant cette « ancienne » typologie de celle que va proposer Blanchard, c'est la valence accordée à la sexualité. Contrairement à la typologie primaire/secondaire, celle de Blanchard accorde une place centrale à la sexualité. Ce qui rapproche ou distingue les transsexuels, c'est d'abord leur orientation sexuelle : il y a les transsexuels homosexuels et les non-homosexuels (hétérosexuels, bisexuels, asexuels). Mais cette différence, exemplifiée pourtant par plusieurs traits, s'explique essentiellement, selon Blanchard, par leur éventuelle inclinaison autogynéphile : les transsexuels androphiles ne sont pas autogynéphiles alors que les autres le sont. Ainsi, la dysphorie de genre non-homosexuelle est explicitée par l'autogynéphilie des sujets. Les transsexuels qui portent un intérêt érotique aux vêtements féminins sont, en réalité, autogynéphiles ; de même que les hétérosexuels qui désirent, en fait, en leur partenaire, la femme qu'ils aimeraient être ; quant aux bisexuels, ils ne sont pas attirés par le corps ou le physique masculins comme le sont les transsexuels homosexuels, mais par la perception d'eux-mêmes en femmes suscitant l'intérêt érotique des 
hommes. En ce sens, leur bisexualité n’est qu’un effet de leur orientation autogynéphile. On comprend que l'autogynéphilie est, selon Blanchard, une paraphilie véritable, qu'on peut définir spécifiquement comme une « déviation d’objet » du désir hétérosexuel masculin . Elle est, en outre, fondamentale pour comprendre le transsexualisme masculin: c'est ce « problème sexuel » qui amène un certain nombre d’hommes biologiques à demander une réassignation.

Ce modèle paraphilique semble s'adapter moins aisément à la situation et à l'expérience des FtM. Dans les années 1980, Blanchard confirme ce dont presque tout le monde était convaincu alors, à savoir que les transsexuelles sont très rarement attirées par les hommes. Dans sa version féminine, le transsexualisme serait donc fondamentalement homosexuel. Pourtant, l'androphilie des FtM, qui va être de plus en plus prise en considération à partir de cette période et surtout dans les années 1990, va conduire à une tentative de typologisation comparable à celle élaborée pour les MtF. Une étude a notamment été menée, par des collaborateurs de Blanchard, sur des femmes biologiques demandant ou ayant réalisé une réassignation et distinguées selon leur orientation sexuelle définie d'après l'échelle de Kinsey. Se référant à un certain nombre d'études qui se sont attachées à établir les caractéristiques de genre et les préférences sexuelles de femmes biologiques hétérosexuelles et homosexuelles, les auteurs ont tenté de différencier les transsexuelles homosexuelles et non-homosexuelles. Les résultats suggèrent que les transsexuelles homosexuelles présentent une plus grande non-conformité de genre dans l'enfance, préfèrent des partenaires plus féminines, expérimentent une jalousie plus sexuelle qu'affective, sont sexuellement plus actives, ont plus de partenaires, ont un plus fort désir de phalloplastie et ont plus d'intérêt pour les stimuli sexuels visuels. En revanche, rien ne les distingue des transsexuelles nonhomosexuelles pour ce qui concerne leur désir général de masculinisation corporelle, ou encore le statut social, l'attractivité et l'âge de leurs partenaires. La conclusion de Chivers et Bailey est assurée bien que prudente: il y a, selon eux, une différence évidente entre transsexuelles homosexuelles et non-homosexuelles, mais il faudrait poursuivre la recherche pour s'assurer de la présence d'une éventuelle autoandrophilie des FtM. Ils ne semblent, cependant, guère optimistes en la matière : " given that the incidence of true paraphilia among genetic females is extremely low (DSM-IV), we would expect that " autoandrophilia » would be rare or nonexistent among nonhomosexual FtMs. ». Autrement dit, la typologie de Blanchard est confirmée de manière plutôt désinvolte : elle semble valoir dans tous les cas de transsexualisme qu'ils soient masculins ou féminins, alors même que ce qui en constitue le noyau fondamental, le fait d'être sexuellement excité par l'image de soi dans l'autre genre, ne semble pas exister dans la version féminine.

De façon générale, on comprend que la théorie autogynéphilique modifie sensiblement la version classique du transsexualisme. Il y a, d'ailleurs, deux manières d'envisager cette modification. Dans une première option, on peut envisager la proposition de Blanchard comme un simple affinement typologique du transsexualisme: il y aurait un type de transsexualisme qui se donnerait plutôt comme un trouble de l'identité sexuée (correspondant au transsexualisme "classique » voire au transsexualisme primaire) et un autre qui serait plutôt un trouble de la sexualité. Dans cette hypothèse, Blanchard aurait seulement caractérisé un sous-type spécifique de transsexualisme, le transsexualisme autogynéphile. Pourtant l'ambiguïté liée à la double expression typologique qu'il propose amène à penser que les deux formes de transsexualisme ont, en réalité, affaire avec des problèmes de sexualité. Dire, en effet, qu'il y a un transsexualisme homosexuel et un transsexualisme autogynéphile (ou nonhomosexuel), c'est suggérer implicitement que tous les types de transsexualisme ont affaire avec la sexualité, c'est-à-dire sont explicitables par son intermédiaire. Il est difficile de savoir dans quelle mesure Blanchard lui-même soutiendrait cette option, mais elle imprègne 
suffisamment sa conceptualisation pour que John Michael Bailey, qui s’est fait récemment le vulgarisateur de sa théorie, la reprenne à son compte: « One cannot understand transsexualism without studying transsexuals'sexuality. (...) Those who love men become women to attract them. Those who love women become women they love.»; ou encore: "Homosexual transsexuals are in their own way just as sexually motivated as autogynephiles. » . Ce livre a déclenché une violente controverse initiée par plusieurs femmes transgenres de renom et relayée dans la communauté «trans » ${ }^{4}$. Une part de la colère qu'a suscité l'ouvrage tient à la présentation irrespectueuse qui est faite des femmes transgenres, mais ce qui rassemble les critiques, qu'ils soient transgenres ou praticiens, c'est la part donnée à la sexualité dans l'explicitation du transsexualisme. Ce qui fâche, ce n'est pas tant que Bailey questionne le rôle de la sexualité dans le transsexualisme, c'est bien plutôt le fait que la dysphorie de genre soit complètement et entièrement considérée comme une pathologie de l'identité sexuelle. Contrairement à ce qu'affirmait Benjamin, le transsexuel n'a plus ici un " gender problem » mais bien un "sexual problem ». En outre, le spectre de l'homosexualité comme paraphilie plane quelque peu ici : même si aucun des auteurs en question ne soutient une conception pathologique de l'homosexualité, les choses sont suffisamment ambiguës et entremêlées pour pouvoir être lues comme telles. Un certain nombre de critiques de Bailey lui reprochent, d'ailleurs, de faire le jeu de la droite homophobe et bigote américaine (à défaut de " guérir » le coupable penchant homosexuel, la réassignation " hétérosexualise » de manière efficiente). Par ailleurs, au moment où beaucoup de personnes transgenres militent pour la dépsychiatrisation de la transsexualité, cette version n'est pas faite pour les rassurer. En ancrant, plus encore, le transsexualisme dans le cadre des « solides » paraphilies, elle réduit considérablement les possibilités de l'exclure de l'univers des pathologies psychiatriques. Enfin, par rapport à la conception pathologique classique, elle fait peser un risque de délégitimation et de restriction sur les traitements. En effet, la normalisation, que la version classique avait gagnée en secondarisant la sexualité, semble ici de nouveau affaiblie. Si Blanchard et Bailey sont convaincus de l'intérêt des opérations, quelle que soit la forme de transsexualisme observée, il n'est pas sûr que tous les praticiens soient prêts à intervenir pour les cas qui seraient diagnostiqués comme autogynéphiles. Nombre de personnes transgenres craignent ainsi d'être socialement et médicalement fragilisées par cette nouvelle version du transsexualisme.

Le modèle paraphilique proposé par Blanchard, en sexualisant totalement et définitivement le transsexualisme (notamment dans sa version forte), réaffirme de fait l'existence d'un lien avéré et nécessaire entre "pathologies sexuées » et "pathologies sexuelles », lien que la psychiatrie et la sexologie avaient pourtant participé ou consenti à dénouer dans le courant du $20^{\text {ème }}$ siècle. Cette conception pourrait infléchir le texte du DSM V dont la sortie est prévue en 2013. Les premières ébauches de cette nouvelle version rendue publique sur le site de l'APA au printemps $2010^{5}$ établissent effectivement un lien entre ces pathologies mais de manière assez subtile. L'esquisse du DSM V utilise les catégories d'autogynéphilie et d'autoandrophilie mais pas directement dans le cadre de ce qui est désormais nommé la Gender Incongruence (GI). Elles sont présentes en fait dans le Transvestic fetichism, et sont envisagées comme un pont entre ce trouble fétichiste et la GI

\footnotetext{
${ }^{4}$ Pour une synthèse de l'histoire de la controverse et des débats qui l’ont accompagné cf . le numéro spécial que lui a consacré la revue Archives of sexual behavior. Kenneth Zucker, ed., Special Issue. The Controversy Surrounding the Man Who Would Be Queen, vol. 37 (Archives of sexual behavior, 2008).

${ }^{5}$ Blanchard est l'un des membres du groupe de travail chargé de la révision des troubles de l’identité sexuée et sexuelle.
} 
puisqu’il est précisé que le type autogynéphilique présente un risque élevé de dysphorie de genre. L'autogynéphilie est donc bien ce qui relie le trouble de l'identité de genre aux paraphilies. On trouve, par ailleurs, dans cette ébauche une reconnaissance de l'inutilité diagnostique de la sexualité mais assortie d'une invitation, paradoxale dans le cadre d'un manuel diagnostique, à la conserver dans la description du nouveau GI en raison de son utilité pour la recherche. Il est enfin signalé que la définition de l'orientation sexuelle des personnes transgenres n'est pas aisée ce qui nous amène au troisième volet de notre exploration.

\section{Définir l'orientation sexuelle, identifier les personnes}

Explorer la manière dont on définit l'orientation sexuelle des transsexuels peut paraître au premier abord sans intérêt dans la mesure où nous savons tous très bien comment l'on définit l'orientation sexuelle d'une personne. Nous savons notamment que nous devons, pour le faire, connaître à la fois le sexe de cette personne et celui des partenaires qu'elle désire sexuellement. Rien de plus simple a priori. Cependant, dans le cas des personnes transgenres, le problème se corse légèrement du fait justement de la transition de genre qu'elles expérimentent. Comme le souligne J. Butler, « si nous ne pouvons nous référer sans ambiguïté au genre dans de tels cas, quel point de référence avons-nous pour affirmer quelle est l'orientation sexuelle d'une personne ?». Essayons de voir comment la psychiatrie et la sexologie s'arrangent avec cette question quelque peu épineuse. Une première solution, généralement utilisée dans la clinique du transsexualisme, est celle prônée par Benjamin, qui consiste à qualifier doublement l'orientation sexuelle de la personne transsexuelle en fonction de la partition sexe psychique/sexe biologique, généralement utilisée dans ce cadre. Ce qui amène à requalifier l'orientation sexuelle après la transition, notamment lorsque celle-ci correspond à une attirance exclusive pour les personnes d'un sexe particulier comme le montre le tableau suivant.

\begin{tabular}{|l|c|c|c|c|}
\hline $\begin{array}{l}\text { Qualification du } \\
\text { DSM }\end{array}$ & MtF & MtF & FtM & FtM \\
\hline $\begin{array}{l}\text { Attirance pour } \\
\text { les hommes }\end{array}$ & Homosexualité & Hétérosexualité & Hétérosexualité & Homost-transition \\
\hline $\begin{array}{l}\text { Attirance pour } \\
\text { les femmes }\end{array}$ & Hétérosexualité & Homosexualité & Homosexualité & Hétérosexualité \\
\hline $\begin{array}{l}\text { Attirance pour } \\
\text { les deux sexes }\end{array}$ & Bisexualité & Bisexualité & Bisexualité & Bisexualité \\
\hline $\begin{array}{l}\text { Attirance ni } \\
\text { pour l'un ni } \\
\text { pour l'autre }\end{array}$ & Asexualité & Asexualité & Asexualité & Asexualité \\
\hline
\end{tabular}

Cette requalification imposée par la transition est cependant essentiellement visible dans la pratique clinique. Dès lors qu'il s'agit de conduire des travaux de recherche sur la sexualité des personnes transsexuelles, les auteurs ont pris l'habitude, comme nous avons pu le voir, de définir l'orientation sexuelle relativement au sexe d'origine entendu comme le sexe biologique. Cette manière de procéder est largement contre-intuitive, voire irrespectueuse 
comme le disait Benjamin, puisque cela oblige, par exemple, à considérer une femme transgenre mariée à un homme, comme un homosexuel ou encore à tenir pour hétérosexuelle un homme transgenre gay. Ce choix tient sans doute aux contraintes liées à la recherche quantitative, notamment à l'obligation de maintenir une certaine cohérence des échantillons au regard de l'orientation sexuelle et particulièrement lorsqu'ils comprennent simultanément des personnes en situation de pré ou de post transition ou encore lorsqu'il s'agit de comparer les mêmes personnes avant et après la transition. Cependant, cette traduction de l'attirance sexuelle reste étonnante car il serait sans doute plus aisé d'utiliser alors les termes androphile ou gynéphile qui sont d'ailleurs parfois évoqués mais qui ne sont jamais utilisés comme des qualificatifs courants, contrairement aux termes hétérosexuel et homosexuel. Cet usage attire l'attention sur le fait qu'on rend équivalentes ici deux manières de définir l'orientation sexuelle qui, bien qu'imbriquées, sont pourtant différentes. En effet, dans une première optique, il est possible de définir l'orientation sexuelle d'une personne en prenant uniquement en considération le sexe du partenaire qu'elle désire. C'est le fameux «attiré par» du DSM qu'on trouve d'ailleurs dans un certain nombre de textes de psychiatres et de sexologues décliné en terme de «-philie». Dans une seconde optique, on peut définir l'orientation sexuelle en prenant en considération à la fois le sexe du «désirant » et le sexe du " désiré »: c’est bien ce que disent les termes homosexualité (partenaires de même sexe), hétérosexualité (partenaires de sexe différent), ou encore bisexualité (sexe des partenaires variable). Ainsi, nous utilisons conjointement une manière " unicentrée » et une manière " comparée » de définir l'orientation sexuelle des personnes : dans le premier cas, les termes employés restent muets sur le genre du désirant et focalisent l'attention sur la qualité sexuée du sujet-objet de désir (gynéphilie, androphilie, autogynéphilie, autoandrophilie, etc.), alors que dans le second, les termes prennent nécessairement en considération le genre des deux partenaires et définissent leur relation en termes d'identité/altérité. L'usage particulier de ces deux modalités de qualification dans les recherches portant sur la sexualité des transsexuels, éclaire les opérations de réduction inhérentes à notre conception de l'orientation sexuelle. Définir l'identité sexuelle d'une personne, c'est en effet d'une part, faire du genre des partenaires sexuels le fondement de l'intérêt qu'ils se portent et, d'autre part, réduire leur engagement sexuel à la combinaison de leur qualité sexuée respective. Pourtant, on conçoit aisément qu'associer l'intérêt sexuel d'une personne au seul genre de ses partenaires est réducteur au regard de l'ensemble des désirs, fantasmes et plaisirs qui l'intéressent. En ce sens, si l'on voulait qualifier de façon plus réaliste l'orientation sexuelle de quelqu'un, il ne faudrait pas seulement, comme le fait le DSM (et comme nous le faisons ordinairement), demander «attiré par qui ? ", mais plutôt «intéressé à quoi avec qui ? ". C’est un peu ce qu'agite Blanchard avec son autogynéphilie ${ }^{6}$ et c'est bien avec une telle question que travaillent les sexologues et les autres praticiens, y compris lorsqu'ils reçoivent des transsexuels. Pourtant dès lors qu'il s'agit de déterminer l'orientation sexuelle d'une personne, le " faire quoi avec » disparaît au profit du seul « qui ». C'est ce qu'on observe dans les recherches portant sur la sexualité des transsexuels, où la détermination de l'identité sexuelle d'une personne, se fonde essentiellement sur le genre de ceux qui «lui font de l'effet ». Parmi les différents modes d'évaluation de cette attirance, la manière la plus simple consiste à se fonder sur les déclarations de la personne elle-même. C'est ce que font, par exemple, ceux qui utilisent l'échelle de Kinsey avant de tracer leurs propres frontières dans les sept degrés qu'elle comporte pour établir qui est homosexuel, bisexuel ou hétérosexuel ${ }^{7}$. Mais assez souvent,

\footnotetext{
${ }^{6}$ S'il « invente » l'autogynéphilie c'est bien à partir de son intérêt à décrire au plus près l'intérêt sexuel de «transsexuels masculins" pour le féminin. Personne d'ailleurs ne songe à nier la valeur descriptive de la notion d'autogynéphilie.

${ }^{7}$ D'une étude à l'autre les catégories ainsi établies ne sont d'ailleurs pas homogènes. Par exemple, Lawrence (2005) classe respectivement les deux degrés les plus extrêmes comme homosexuel et
} 
cette simple déclaration semble insuffisante et l'on met au point des échelles ad hoc qui questionnent les désirs et les pratiques de manière plus détaillée comme le fait, par exemple, Blanchard avec ses échelles d'androphilie et de gynéphilie qui comportent chacune de nombreux items. Parfois aussi, les recherches passent du questionnaire plus ou moins élaboré à la mesure instrumentale de l'excitation sexuelle : l'émoi des sujets, confrontés à des stimuli visuels, est évalué par l'intermédiaire de la pléthysmographie pénienne ou de la photopléthysmographie vaginale ${ }^{8}$. Ce genre d'investigation poussée n'a pas pour objectif d'obtenir une description plus complexe des désirs et des attachements sexuels des personnes car elle sert essentiellement à qualifier l'orientation sexuelle grâce au seul genre du " qui » excitant. Il y a plutôt là, semble-t-il, une volonté de " déjouer » les réponses stratégiques et conformistes qui, bien évidemment, constituent autant de biais pour l'interprétation des résultats, mais aussi de détecter la "véritable » orientation sexuelle du sujet qui peut luimême méconnaître la réalité de son désir. C’est ce que suggère, entre autres, Anne Lawrence, dans la critique d'une étude qui remet en question les résultats de Blanchard : " many of these cases are probably MF transsexuals who have not described their sexual orientation accurately. It is widely recognized that some nonhomosexual MF transsexuals inaccurately describe themselves as homosexual, occasionally in attempt to deceive clinicians, but more commonly because they misinterpret sexual arousal to the idea of being a woman having sex with a man as sexual arousal to the male somatotype. " (Lawrence 2008, 319). Il y a donc ici l'idée d’une vérité du désir érotique, et donc de l’identité sexuelle, qui se situe au-delà de la déclaration des personnes et dont la mesure instrumentale serait, en dernière instance, le moyen idéal d'investigation. On comprend que ces modes de définition et d'évaluation renvoient à une conception essentialiste de l'orientation sexuelle qui non seulement est considérée comme une propriété constitutive et stable des personnes mais aussi comme étant biologiquement fondée. On n'est ainsi guère étonné de constater qu'un certain nombre de chercheurs qui s'intéressent à la sexualité des transsexuels mènent parallèlement des investigations sur les liens entre homosexualité et longueur des doigts de la main ou encore entre homosexualité et rang de naissance dans la fratrie ; ou bien qu'ils interprètent les changements d'orientation sexuelle observés parfois après la transition ${ }^{9}$ sur le versant de l'erreur d'évaluation. Il est, en effet, moins couteux d'expliciter une modification observée de l'attirance sexuelle par un recours au mensonge ou à la méconnaissance de la "véritable » orientation, comme le fait Lawrence, que de développer une théorisation élaborée des facteurs biologiques qui pourraient en rendre compte.

Quoi qu'il en soit, on a le sentiment que les investigations sur la sexualité des transsexuels sont plus particulièrement utilisées pour asseoir la conception socialement dominante de l'orientation sexuelle que pour véritablement la questionner alors même que c'est ce qu'elle nous invite à faire. Quand l'appartenance sexuée est discutée et que les corps

hétérosexuel et les trois degrés médians comme bisexuels, alors que Chivers et Bailey (2000) distinguent seulement deux classes, les homosexuels (4 premiers degrés de l'échelle) et les nonhomosexuels (les trois autres degrés).

${ }^{8}$ Ces techniques consistent à mesurer l'augmentation du volume sanguin. Bien que leur validité soit controversée dans l'évaluation de l'orientation sexuelle des individus, elles sont utilisées pour déterminer diverses paraphilies dont notamment la pédophilie. Dans le cadre du transsexualisme, elles ont été utilisées par Blanchard et certains de ses collaborateurs. Cf. notamment Anne Lawrence et al., "Measurement of Sexual Arousal in Postoperative Male-to-Female Transsexuals Using Vaginal Photoplethysmography," Archives of sexual behavior 34, no. 2 (2005).

${ }^{9}$ Christopher Daskalos, "Changes in the Sexual Orientation of Six Heterosexuals Male-to-Female Transsexuals," Archives of sexual behavior 27, no. 6 (1998).; Peter Bentler, "A Typology of Transsexualism : Gender Identity Theory and Data," Archives of sexual behavior 5, no. 6 (1976). 
sont travaillés de conserve n’y a-t-il pas justement une possibilité d'envisager autrement l'identification sexuelle des personnes? Ainsi lorsqu'un FtM dit, au praticien qui le reçoit pour une demande de transition: «j'aime les femmes mais ça n'a rien à voir avec l'homosexualité » ou encore lorsqu'un autre (qui a réalisé une transition) affirme à Holly Devor, que la relation qu'il a avec sa partenaire féminine est une relation homosexuelle masculine ${ }^{10}$, est-il possible de comprendre ce qui est dit sans avoir recours aux stratégies de requalification ou de contournement observées? Suspendons un instant nos réflexes identificatoires pour voir ce qu'il en est. En fait, comprendre ce qui est affirmé là, suppose de s'attacher à qualifier les engagements et les actions plutôt que les individus, c'est-à-dire à définir l’identité/altérité de leurs enrôlements sexuels plutôt que l'identité/altérité de leurs corps et de leurs genres d'appartenance. Autrement dit, ce qui importerait pour saisir, au plus près, l'orientation sexuelle d'une personne, serait la manière dont elle s'engage dans la relation, c'est-à-dire la posture sexuelle qu'elle investit comme désirable et source de plaisir. Si tel est le cas, au lieu de parler d'homosexualité lorsque les corps et les genres des partenaires sont semblables, d'hétérosexualité lorsqu'ils sont différents ou encore de bisexualité lorsqu'ils sont dans un rapport variable, il faudrait peut-être réserver ces mêmes termes pour qualifier respectivement les relations où les partenaires jouent sur l'identité, l'altérité ou l'alternance des postures sexuelles qu'ils assument. Cette qualification des engagements ne nous est pas totalement étrangère : on s'en approche lorsqu'on utilise, par exemple, les termes passif/actif, pénétrant/pénétré, top/bottom, pour déterminer plus spécifiquement les implications de chacun. Mais assez souvent, ces catégories identificatoires, du moins les deux premières paires ${ }^{11}$, sont habituellement utilisées pour qualifier l'implication des partenaires de même sexe et semblent avoir pour objectif ou pour effet «d'hétérogenrer » la relation (à défaut d'être homme et femme, on ferait l'homme vs la femme) comme si l'altérité des genres devait nécessairement constituer le point focal de toute sexualité. Or c'est bien justement cette corrélation que la sexualité transsexuelle nous oblige à interroger. En nous invitant à délier les associations ordinaires et à complexifier les implications sexuelles, elle nous engage, en fait, à reconsidérer la qualification sexuelle des personnes. On comprend, cependant, qu'une définition de l'orientation sexuelle des individus qui s'attache plus à leurs attributions qu'à leurs attributs nous oblige à renoncer à une conception identitaire de l'implication sexuelle qui nous est, non seulement familière, mais aussi, assez souvent, commode. Il ne faut pas s’étonner, alors, que les capacités et les volontés individuelles et collectives à s'impliquer dans une telle remise en question se montrent diversement empressées. Quoi qu'il en soit, les pratiques et les approches psychiatriques et sexologiques que nous avons examinées ici, nous invitent à comprendre, que l'enjeu d'un tel réexamen ne renvoie pas simplement à une élaboration plus rigoureuse de la caractérisation des personnes mais surtout aux possibilités d'existence que les catégories produites et adoptées peuvent leur offrir. Au fond, l'important n'est pas tant ce que les catégories disent des gens que ce qu'elles leur font et font faire.

\footnotetext{
${ }^{10}$ Voir également Pat Califia, homme transgenre, ex-lesbienne, qui loin de se considérer comme bisexuel-le écrit dans un texte de 1983: "j’ai des rapports sexuels avec des pédés. Et je suis lesbienne » Pat Califia, Sexe Et Utopie (Paris: La Musardine, 2008).

${ }^{11}$ La distinction top/bottom (dominant/dominé sexuels) est principalement utilisée par les partenaires de pratiques SM et ne comporte pas nécessairement d'effet « d’hétérogenrisation ».
} 


\section{Références bibliographiques}

American Psychiatric Association. Mini Dms-Iv-Tr. Critères Diagnostiques. Version Française. Paris: Masson, 2004.

Bailey, John Michael. The Man Who Would Be Queen: The Science of Gender-Bending and Transsexualism. Washington DC: Joseph Henry Press, 2003.

Benjamin, Harry. "The Transsexual Phenomenon." edited by Symposion Publishing. Düsseldorf: http://www.symposion.com/ijt/benjamin/index.htm, 1966. Reprint,

Bentler, Peter. "A Typology of Transsexualism : Gender Identity Theory and Data." Archives of sexual behavior 5, no. 6 (1976): 567-84.

Blanchard, Ray. "The Classification and Labeling of Nonhomosexual Gender Dysphorias." Archives of sexual behavior 18, no. 4 (1989): 315-34.

. "Early History of the Concept of Autogynephilia." Archives of Sexual Behavior 34, no. 4 (2005): 439-46.

"Fraternal Birth Order and the Maternal Immune Hypothesis of Male Homosexuality." Hormones and Behavior 40, no. 2 (2001): 105-14.

—. "Nonhomosexual Gender Dysphoria." Journal of sex research 24 (1988): 188-93. and Marital Therapy 17 (1992): 235-51.

_. "Typology of Male-to-Female Transsexualism." Archives of sexual behavior 14, no. 3 (1985): 247-61.

Blanchard, Ray, Leonard Clemmensen, and Betty Steiner. "Heterosexual and Homosexual Gender Dysphoria." Archives of sexual behavior 16, no. 2 (1987): 139-52.

Blanchard, Ray, and Richard Lippa. "Birth Order, Sibling Sex Ration, Handedness and Sexual Orientation of Male and Female Participants in a Bbc Internet Research Project." Archives of sexual behavior 36, no. 2 (2007): 163-76.

Butler, Judith. Défaire Le Genre. Paris: Editions Amsterdam, 2006.

Califia, Pat. Sexe Et Utopie. Paris: La Musardine, 2008.

Chivers, Meredith, and John Michael Bailey. "Sexual Orientation of Female-to-Male Transsexuals: A Comparison of Homosexual and Nonhomosexual Types." Archives of sexual behavior 29, no. 3 (2000): 259-78.

Daskalos, Christopher. "Changes in the Sexual Orientation of Six Heterosexuals Male-toFemale Transsexuals." Archives of sexual behavior 27, no. 6 (1998): 605-14.

Devor, Holly. "Sexual Orientation Identities, Attractions, and Practices of Female-to-Male Transsexuals." Journal of sex research 30, no. 4 (1993): 303-15.

Ekins, Richard. "Science, Politics and Clinical Intervention: Harry Benjamin, Transsexualism and the Problem of Heteronormativity." Sexualities 8, no. 3 (2005): 306-28.

Freund, Kurt, Betty Steiner, and S Stan. "Two Types of Cross-Gender Identity." Archives of sexual behavior 11, no. 1 (1982): 49-63.

Lawrence, Anne. "Male-to-Female Transsexual Subtypes : Sexual Arousal with CrossDressing and Physical Measurements." Psychiatry Research 157 (2008): 319-20.

Lawrence, Anne, Elizabeth Latty, Meredith Chivers, and John Michael Bailey. "Measurement of Sexual Arousal in Postoperative Male-to-Female Transsexuals Using Vaginal Photoplethysmography." Archives of sexual behavior 34, no. 2 (2005): 135-45.

Meyerowitz, Joanne. How Sex Changed. A History of Transsexuality in the United States. Cambridge, Massachusetts: Harvard University Press, 2002.

Zucker, Kenneth, ed. Special Issue. The Controversy Surrounding the Man Who Would Be Queen. Vol. 37: Archives of sexual behavior, 2008. 
Zucker, Kenneth, Ray Blanchard, and Marvin Siegelman. "Birth Order among Homosexual Men." Psychological reports 92, no. 1 (2003): 117-18. 